\title{
A Comparative Evaluation of the Intensities, Spectral Resolution, and Overall Time of Acquisition Achievable by SEM-based Parallel Beam WDS and SEM-based Rowland Circle WDS
}

\author{
Stephen M. Seddio ${ }^{1 *}$
}

1. Thermo Fisher Scientific, Surface Analysis and Microanalysis, Madison, Wisconsin, USA.

* Corresponding author: Stephen.Seddio@ThermoFisher.com

Wavelength-dispersive spectroscopy (WDS), when applied to X-ray microanalysis, can achieve excellent quantitative results, even for elements at trace concentrations, and can achieve excellent spectral resolution. Such performance is owed to the use of a diffractor to only detect X-rays of a single energy. Traditionally, WDS spectrometers rely on a Rowland circle geometry and are mounted on an electron microprobe-an electron microscope specifically designed for WDS. An electron microprobe typically has 5 Rowland circle WDS spectrometers which can acquire data concurrently.

WDS spectrometers have also been mounted on scanning electron microscopes (SEM), which are specifically designed for electron imaging. Typically, only one WDS spectrometer is mounted on an SEM. The traditional Rowland circle WDS approach has been applied to SEMs. However, most modern WDS spectrometers available for SEMs rely on a parallel beam geometry [1]. These parallel beam WDS spectrometers rely on a collimating optic inserted near the sample. As well as removing the need for the Rowland circle geometry, the collimating optic, particularly a hybrid collimating optic [1], acquires $\mathrm{X}$-rays with a large solid angle.

Comparative work has been done evaluating the X-ray intensities and spectral resolution achieved on Rowland circle WDS spectrometers on an electron microprobe and a parallel beam WDS spectrometer with a hybrid collimating on an SEM [2]. However, no such comparative evaluation has been done for a Rowland circle WDS spectrometer on an SEM and a parallel beam WDS on an SEM.

For presentation, intensity, spectral resolution, and overall analytical time measurements will be made using a commercially-available $210 \mathrm{~mm}$ radius Rowland circle WDS spectrometer on an SEM and a commercially available parallel beam WDS spectrometer with a hybrid collimating optic (i.e., a Thermo Scientific MagnaRay WDS spectrometer). The samples will be the same elemental and oxide standard samples. The accelerating voltages and beam currents for each analysis will be made to be identical between the two acquisitions. Working distances will be those specified by the WDS and SEM manufacturers. Furthermore, the WDS intensity results will be compared to the calculated intensities for each spectrometer based on calculations of solid angle and X-ray reflectance and transmittance experienced in each spectrometer [3, Fig. 1].

References:

[1] Seddio, S. Intro. To Princ. And Appl. Of Parallel Beam WDS (2014), Thermo Fisher Scientific WP52608.

[2] Seddio, S. and Fournelle, J.H., Micros. Micro 21 (2015), p. 1877.

[3] Seddio, S., EPMA2016 Procedings (2016). 


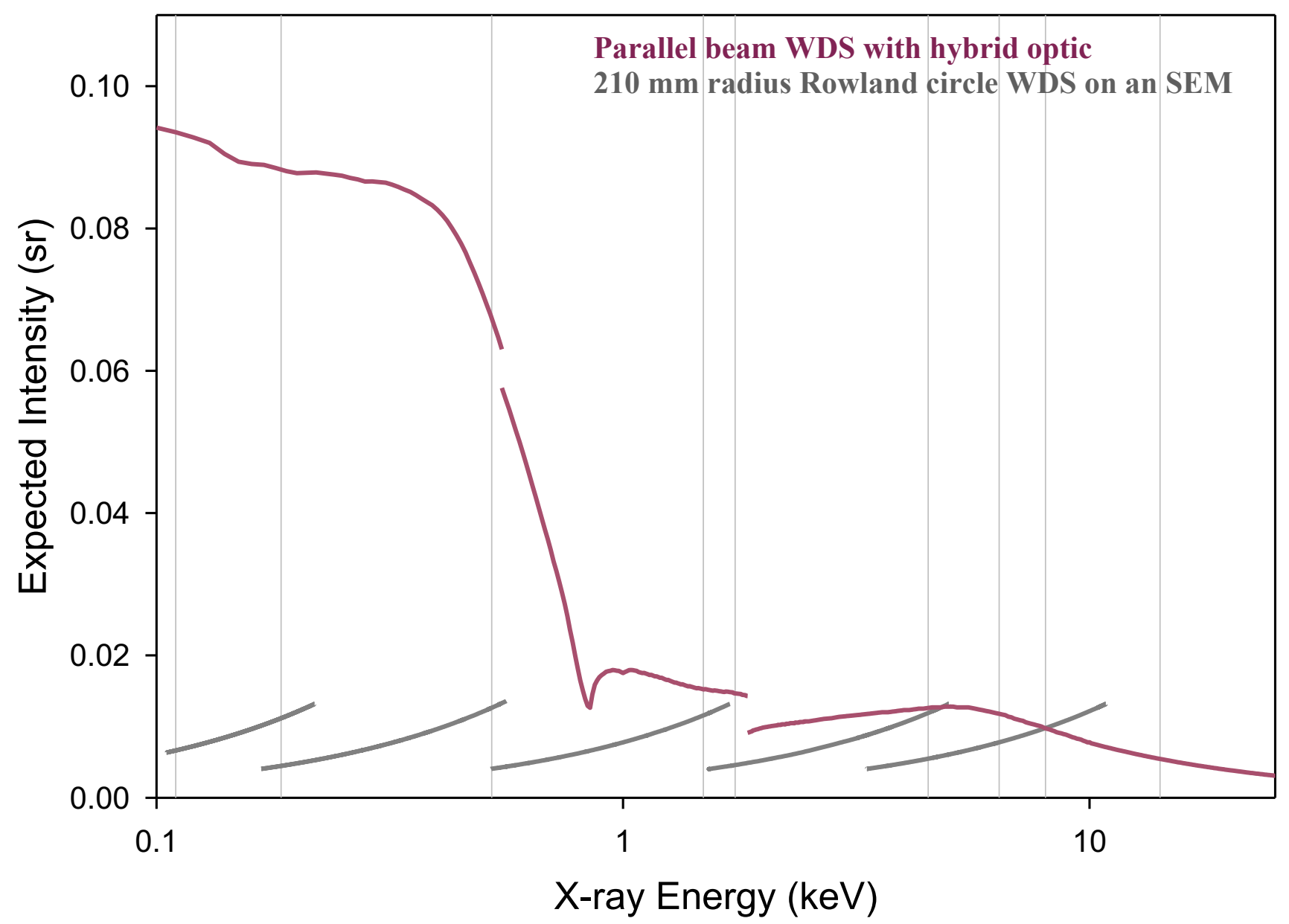

Figure 1. Calculated expected X-ray intensities as a function of X-ray energy. "Expected intensity" is calculated as a measure of the X-ray intensity observed by the diffractor. It is the solid angle of the diffractor or the solid angle of the collimating optic (all collimated X-rays are incident upon the diffractor) which is then scaled by the appropriate transmissivity or reflectance of the optic. 